\title{
Generation and applications of CT images of the ideal standing position for total knee arthroplasty surgery
}

\author{
A. Doi ${ }^{1}$, H. Takahashi ${ }^{1}$, T. Mawatari ${ }^{2}$ \& S. Ichinohe ${ }^{3}$ \\ ${ }^{1}$ Iwate Prefectural University, Japan \\ ${ }^{2}$ Hamanomachi Hospital, Japan \\ ${ }^{3}$ Iwate Medical University, Japan
}

\begin{abstract}
CT images with body weight loading are desired for preoperative planning on TKA surgery. In our proposed method, we perform stress analysis by the finite element method (FEM) for bone mesh structure by generating CT images, and we estimate an ideal standing position of the loaded state (referred to as deformed CT images of the ideal standing position). We also compare the deformed CT image with CR images, and evaluate the effectiveness in several ways.
\end{abstract}

Keywords: conventional preoperative planning, computed radiography (CR), computed tomography, finite element model (FEM).

\section{Introduction}

One problem associated with population aging is the rapid increase in the occurrence of knee joint diseases, treatment for which includes artificial knee joint replacement (total knee arthroplasty (TKA)) and osteotomy. Conventional preoperative planning uses inferior limb X-ray images (two-dimensional computed radiography (CR) images) in the standing position (with body weight loading). Because inferior limb CR images acquired in the standing position provide the information required for preoperative planning, such as the loading stress experienced by the patient in everyday life, it is advantageous for estimating the corrected loading stress after artificial knee joint implant. It is difficult, however, to determine the precise placement of the artificial joint, since front- and side-view CR images are acquired separately. In comparison with CR 
images, computerized tomography (CT) images provide precise and consistent information for each patient, but they are acquired in a lying position, and thus the resulting images do not provide a full account of body weight loading. Therefore, the CT image with body weight loading is desired for preoperative planning on TKA. In order to achieve such a CT image, we convert CT image to CT image of body weight loading by using stress analysis. The deformed CT image has the merits of both CR and CT images. In our approach, we generate iso-surfaces of bone from CT images, and convert them into a tetrahedral cell mesh. Next, we apply a stress analysis, which fix the heel positions and apply stress on the pelvis to deform the inferior limbs. In our experience, we utilize three commercial software products. Volume Extractor Ver.3.0 [1] is used for segmentation and reconstruction of the bone polygons, and FreeForm FFM Ver. 10 [2] for converting them into surface data, and CATIA Ver. 5.0 R20 [3] for generating the tetrahedral cell mesh and the stress analysis [4]. Finally, we compare the deformed CT image with CR images, and evaluated the effectiveness in several ways.

\section{Data acquisition and mesh generation}

\subsection{Overview of volume extractor}

Volume Extractor (VE) is a practical 3D image processing software product, which was researched and developed at Iwate Prefectural University, Japan. Generally, isographic data generated from medical images contains imperfections (infinitesimal holes, surface inversions, etc.) due to the presence of unnecessary shapes and noise, and it necessary to remove these imperfections in order to generate output using a 3D printer or a finite element method (FEM). Figure 1 shows an example of constructing a 3D image from multiple 2D images and the approximation of its surface using 3D polygons.

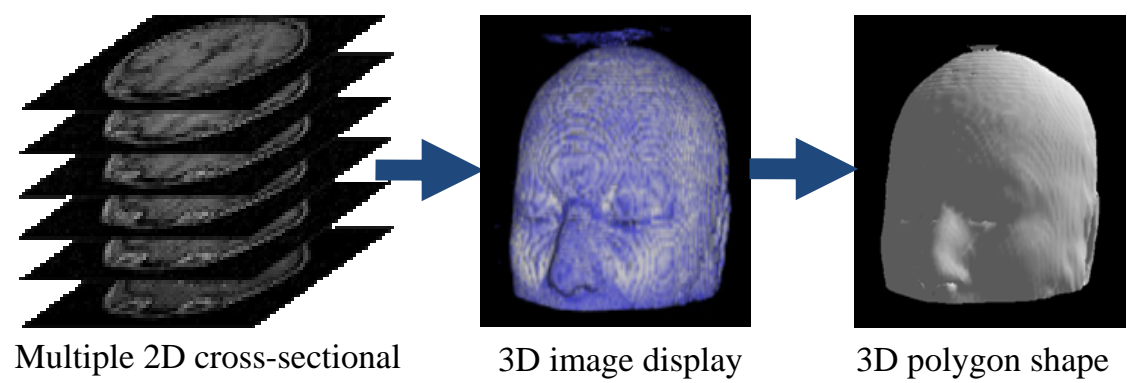

MRI images

Figure 1: $\quad$ Construction of 3D images and reconstruction of shapes.

The software is compatible with reading MR and CT medical images in the general DICOM image format. Furthermore, it is also compatible with image data in TIFF, BMP and RAW formats. In VE, it is possible to display multiple 3D images at the same time. For example, it is possible to display CT images 
which are used for observing the shape of bone alongside with MRI images which are used for observing cartilage and internal organs. Only the 3D image containing the part for extraction is cut (clipped) from the 3D image obtained by CT or MR imaging. Furthermore, by utilizing relevant editing techniques, any unnecessary areas or areas containing artifacts are removed from the clipped 3D image. The software supports cross-section, iso-surface, and volume rendering display methods, as well as any combination of the three.

\subsection{Extraction and editing of the necessary parts}

By using the classification segmentation function based on histograms, the brightness of all areas apart from those corresponding to the bone is set to a specific value (e.g., zero), thus leaving only the brightness values for the bone. The image filter of VE incorporates garbage removal and smoothing functions. In general, the boundary between the cartilage and the bone is not clear, and furthermore, it is necessary to remove the structures corresponding to muscle and tendon. For this purpose, the present function regards the surface of the bone as smooth and presents a clear image of the bone boundary. In case it is necessary to close the surface of the clipped image, by using relevant editing functions, the clipped surface is given the same brightness value as that of the extracted bone. If iso-surfaces are generated by applying the following process, the cross-section of the generated surfaces crosses the surface of the bone, thus forming a closed shape. The 3D shape is reconstructed by taking the brightness values representing the bone boundary as iso-surfaces. In case the amount of iso-surface data is too large, the 3D image data is compressed, and either iso-surfaces are generated or the generated iso-surfaces (polygon data) are deleted. Although the processing of compressed 3D image data is fast, the surface shape is broken. For this reason, it is possible to generate shapes with higher accuracy if the 3D image data is not compressed and the generated iso-surface data is processed with the polygon data removal algorithm [5].

\subsection{Generation of tetrahedral cell mesh for stress analysis}

The generated iso-surfaces are subjected to minor adjustments by using the automatic removal function of the mesh editing function, the automatic rotation function, and the automatic group division function. In the case of human CT and MR images, infinitesimal gaps are generated due to noise or in the process of iso-surface generation. For this reason, it is necessary to examine the adjacent edges and automatically repair gaps with small areas. Furthermore, by subsequently using the information regarding the vectors normal to adjacent surfaces, automatic rotation is performed (correction of the inverted surface) in case there are contradictions in the direction of the surface. Finally, the entire iso-surface data is spatially divided into groups, and any small groups which are spatially disconnected are automatically removed from the groups attributing to the structure. The generated iso-surfaces of bone structure parts are outputted in the external file in STL (Standard Triangulated Language) data format. Next, the STL data is converted into surface data by using the FreeForm software product. 
Finally, the surface data is converted into tetrahedral cell mesh for stress analysis by using CATIA.

\section{Analysis}

Mechanical property values were input into the constructed finite element model (FEM). The boundary condition was applied, followed by the calculation of an analysis solver of CATIA Ver. 5 R20. Analysis type was linear elastic stress analysis, and 3D tetrahedron and pentahedron were employed. The hardware specification of our personal computer is DELL precision M6400 Intel Core2 Quad, 2.26GHz, 7.98GB RAM with Nvidia Quadro Fx 27000M graphics card. Figure 2 shows the generated mesh structures and the results of the analysis. The yellow arrows at the pelvis show the stress and the direction that the strength of body weight is applied, and the blue lines at knee show the fixed nodes. The gap between femur and tibia is connected interactively by using general analysis connection function that is supported by CATIA Ver.5.0. This function produces a joint relationship between femur and tibia parts virtually. The friction type was Coulomb friction, and the friction coefficient $\mu$ was set at 0.001 .

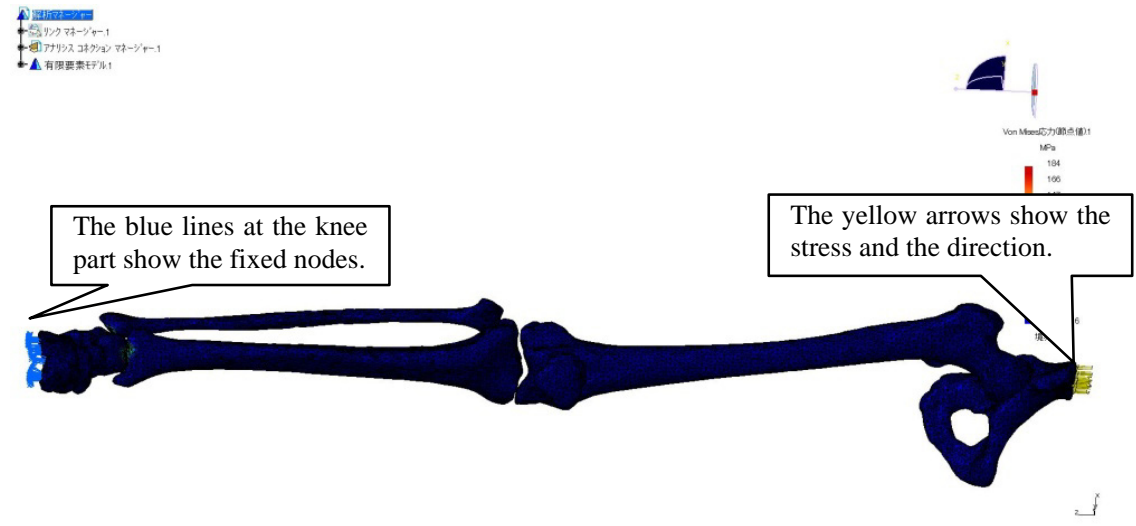

Figure 2: $\quad$ Constraining and loading conditions.

\section{Evaluation and results}

The CT images used in the evaluation were obtained from three healthy male subjects (aged 54, 66, and 69 years). The respective resolutions and the pixel pitch of the images for each subject are presented in Table 2. Figure 3 shows an overall view of the FEM model. Each model of pelvis, femur, tibia, and heel was constructed. The number of element, nodal point, and tetrahedral cell are shown in Table 3. The TE4 shows linear tetrahedral cell, and TE10 shows quadratic tetrahedral cell. Table 4 shows components and mechanical properties of the model. Figure 4 shows the displacement of simulation results for the stress analysis. A total of 11.76, 11.76, and $14.70 \mathrm{GPa}$ of vertical loads to the occlusal plane was applied on the pelvis. The maximum displacement is $10.258 \mathrm{~mm}$, and 
Table 1: $\quad$ Resolution of CT images.

\begin{tabular}{|l|l|l|l|}
\hline ID & Part & Resolution [px] & Pitch [mm] \\
\hline A & Right leg & $512 \times 512 \times 1499$ & $(0.416,0.416,0.63)$ \\
\hline B & Right leg & $512 \times 512 \times 1595$ & $(0.416,0.416,0.63)$ \\
\hline C & Both leg & $512 \times 512 \times 1501$ & $(0.416,0.416,0.63)$ \\
\hline
\end{tabular}

Table 2: $\quad$ The number of elements, nodes, and tetrahedral cells.

\begin{tabular}{|l|l|c|c|c|c|}
\hline ID & Nodes & Elements & Cells (total) & Tet. 4 & Tet. 10 \\
\hline A & 65875 & 238479 & 238437 & 238437 & 0 \\
\hline B & 23765 & 95281 & 94763 & 94763 & 0 \\
\hline C & 112404 & 102826 & 101044 & 38927 & 62117 \\
\hline
\end{tabular}

Table 3: $\quad$ Components and mechanical properties

\begin{tabular}{|l|l|c|c|}
\hline ID & Sex & Young's Modulus (GPa) & Poisson's ratio \\
\hline A & Male & 11.76 & 0.30 \\
\hline B & Male & 11.76 & 0.25 \\
\hline C & Male & 14.70 & 0.25 \\
\hline
\end{tabular}
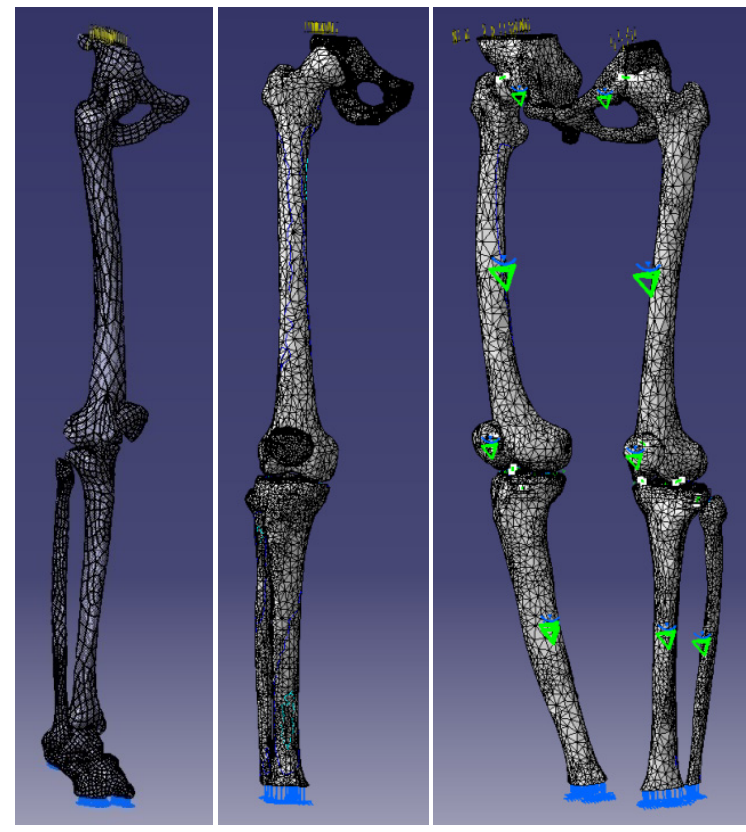

Figure 3: $\quad$ The FEM models (A, B, and C).

the maximum stress value is $2.224 \mathrm{~N} / \mathrm{m}^{2}$. The total time of generating each mesh was about 4 hour, and the computation time of the analysis was about twenty minutes. Evaluation of simulation results of stress analysis is carried out by comparing CR images of the standing position (front view, side view). To evaluate our approach, we acquire front- and side-view CR images (for left and right inferior limbs) and inferior limb CT images of 3 healthy participants. 
Figure 5 illustrates the positions for taking CR images (standing position) and CT images (lying position). Figure 6 shows the front view CR image of right leg, side view CR image of right leg, simulated Model of right leg, and front view CR image of left leg for B. Although our stress analysis considers bone structure only without regard to muscles, tendons, and so on, the deformed bone shapes fit on each CR image roughly.
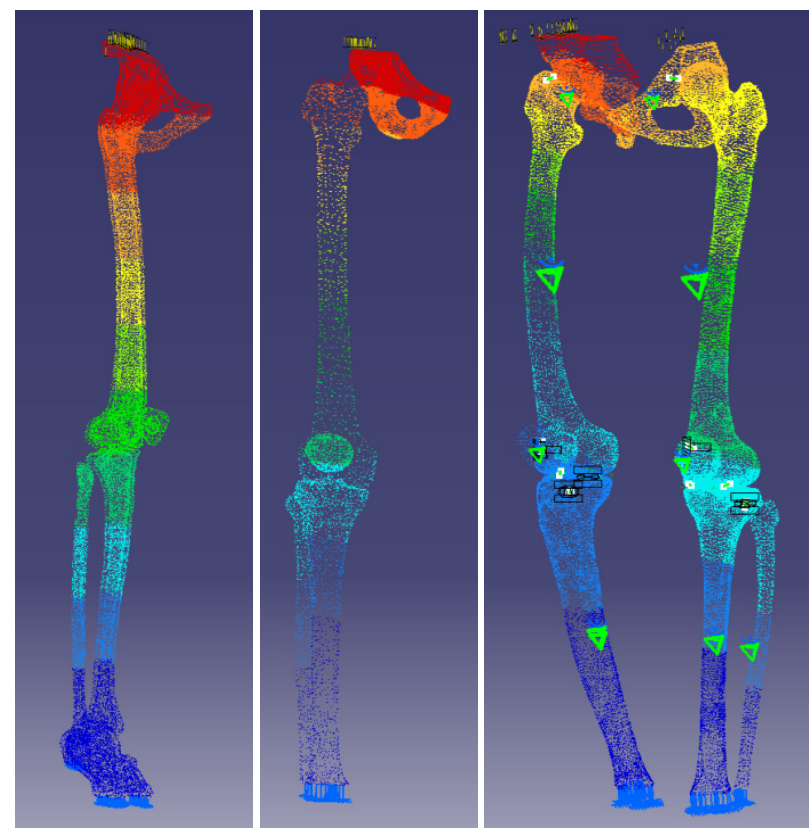

Figure 4: $\quad$ Displacement of each FEM model (A, B, and C).

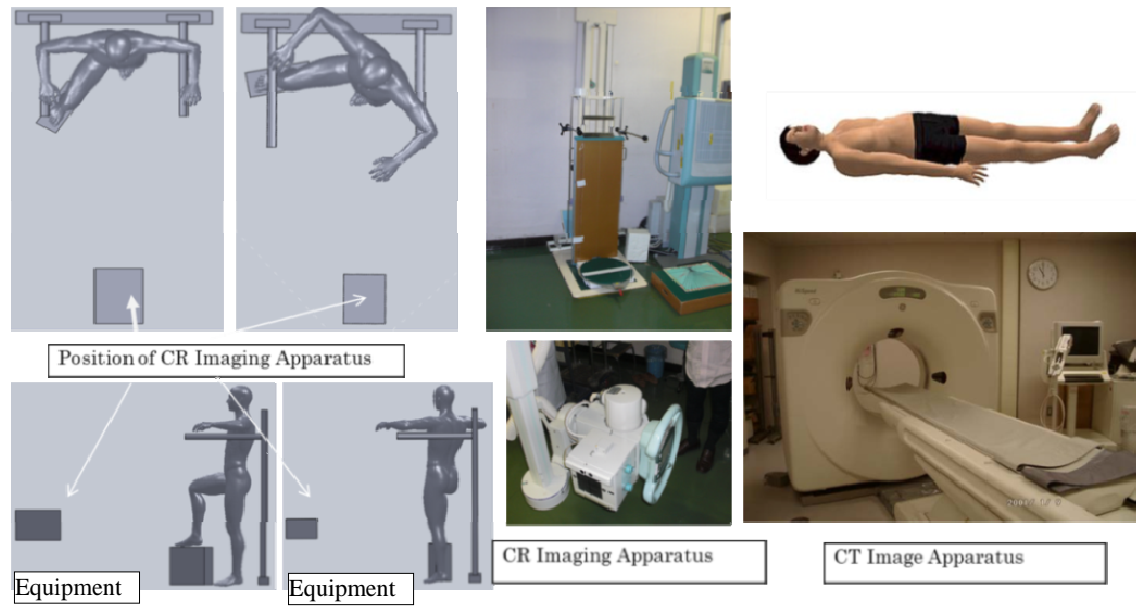

Figure 5: $\quad$ CR/CT imaging equipment for standing and lying positions. 

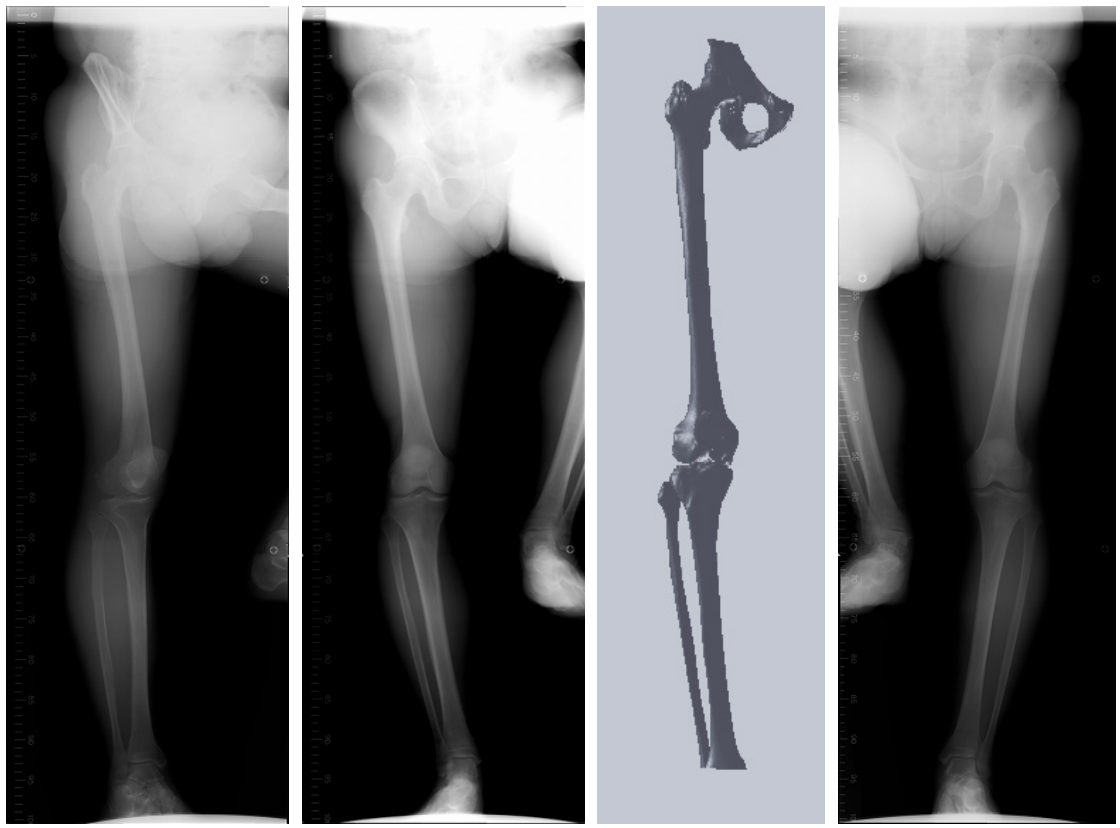

Figure 6: $\quad$ Front view CR image of right leg, side view CR image of right leg, simulated model of right leg, front view CR image of left leg for B.

\section{Conclusion}

We have been investigating the influence of the body weight for the lie down lower-legs that have captured by CT image. The effect of the body weight was examined, and the availability of ideal standing position of lower-legs CT image was proposed in this study. We generated the 3D lower-legs shapes with an ideal standing position by using the mesh generation technique from CT image and the stress analysis by FEM. The conversion between 3D lower-legs shapes and the voxel model can be archived by the several voxel modeling techniques $[6,7]$. The deformed CT image has the merits of both CR and the original CT images. Therefore, the CT image with body weight loading is desired for preoperative planning on TKA.

We have been developing pre-operative planning system for TKA and THA based on the deformed CT images. Our approach allows for precise preoperative planning for TKA [8-10]. It is very useful for determining the exact placement of an artificial knee joint. The exact placement will reduce the burden on doctors and patients, and it will decrease the number of X-ray images taken in which the patient assumes several different positions.

We have also presented the mesh generation techniques using VE Ver. 3.0, with detailed explanation of the concept and the operation of the shape reconstruction functions. The $3 \mathrm{D}$ models prepared with the aid of VE can be utilized in many fields of medicine and engineering [11]. For example, shape 
models containing internal organs, bones, and blood vessels can be used for preoperative planning, operation simulations, medical education, using virtual reality (VR) to obtain informed consent from patients, and so on.

\section{Acknowledgements}

Part of this research was conducted with a Grant-in-Aid for Scientific Research (Project No. 20500425) and the Regional Innovation Cluster Program of Ministry of Education, Culture, Sports, Science and Technology, the A-Step Research Fund of the Japan Science and Technology Agency, and the Iwate Prefectural University Academic Research Fund. Part of this research was conducted with a Grant-in-Aid for Strategic Information and Communications R\&D Promotion Programme (SCOPE) of Ministry of Internal Affairs and Communications. The authors thank Mr. Sachio Kurose, Mr. Masato Tamura, and Mr. Teruki Obara from Iwate Digital Engineer Training Center for their support and help with designing the tailor-made plates.

\section{Reference}

[1] i-Plants Systems, Ltd, "Volume Extractor Ver.3.0", http://www.iplants.co.jp/hp/download/, 2013.

[2] Sensable Technology Ltd., "3D Design and Modeling”, http://www.sensable.com/industries-design-model.htm\#FreeForm, 2013.

[3] Dassault Systems Ltd., "3D CAD design software CATIA", http://www.3ds.com/products/catia/welcome/, 2013.

[4] D. S. Barker et al. Validation of a finite element model of the human metacarpal, Medical Engineering \& Physics 27 pp. 103-113, 2007.

[5] M. Tokuda, A. Doi, F. Itoh, M. Suzuki, H. Haneishi, "An Automatic Extraction of the Bone Axes of Femur and Tibia of Knee Joint Using Inferior limb X-ray Image”, Journal of the Institute of Image Electronics Engineers of Japan, Vol. 37, No. 4, pp. 8-20, 2008.

[6] I. Fujishiro, M. Okutomi, et al. "Visual Information Processing Introduction of CG/Image Processing", CG-ARTS Association, pp. 115116, 2007.

[7] Charles D. Hansen and Chris R. Johnson, "The Visualization Handbook", Academic Press, 2005.

[8] A. Doi, H. Takahashi, S. Mega, and T. Mawatari, "Volume manipulation using cut and paste operations and its applications”, JSST 2012, Int. Conf. on Simulation Technology, Kobe, Sep. 27-28, 2012.

[9] A. Doi, H. Takahashi, and T. Furukawa, "3D Bone Axis Analysis Method Using CT Images of the Lower Limbs and Its Evaluation”, 11th Asian Symposium on Visualization, Niigata, Japan, 2011.

[10] A. Doi, T. Takahashi, T. Mawatari, Sachio Mega, "Development of Volume Rendering System Using 3D Texture Display Techniques and Its Applications”, Medical Imaging Technology, Vol. 30, No. 2, pp. 83-91, 2012. 
[11] A. Doi, H. Takahashi, B. Shuto, M. Katayama, H. Nagashima, M. Okumura, "Design and application of tailor-made plates for treating fractures in small animals", IEEE iCAST 2012, The 4th Int. Conf. on Awareness Science and Technology, Korea, Seoul, Aug. 21-24, 2012. 